\title{
Quantitative Organ Enlargement Assessment
}

National Cancer Institute

\section{Source}

National Cancer Institute. Quantitative Organ Enlargement Assessment. NCI Thesaurus.

Code C135507.

A quantitative assessment of an increase in org an size or dimension. 